\title{
Time-variant linear pulse processing
}

\author{
Haldun M. Ozaktas ${ }^{a}$, Martin C. Nuss ${ }^{b}$ \\ a Bilkent University, Department of Electrical Engineering, 06533 Bilkent, Ankara, Turkey \\ b Bell Laboratories, Lucent Technologies, 101 Crawfords Corner Rd., Holmdel, NJ 07733-3030, USA
}

Received 21 February 1996; revised version received 24 May 1996; accepted 24 May 1996

\begin{abstract}
Previously suggested systems for linear processing of temporal pulses are limited to time-invariant (convolution-type) operations. Although these are the most general operations possible with passive components, we show that by using nonlinear optical interactions, arbitrary linear operations can be performed. Such operations may be useful for performing time-variant analog signal processing, temporal matrix-vector multiplication, and time-slot interchange of pulses for digital communications systems.
\end{abstract}

The most general linear operation $\mathcal{L}$ on a temporal input pulse $f(t)$ yielding the output pulse $g(t)$ can be characterized by the kernel $h\left(t, t^{\prime}\right)$ in the form

$g(t)=\mathcal{L}[f(t)]=\int_{-\infty}^{\infty} h\left(t, t^{\prime}\right) f\left(t^{\prime}\right) \mathrm{d} t^{\prime}$.

It might be helpful to note that the discrete counterpart of this linear superposition is a matrix-vector multiplication of the form

$g_{m}=\sum_{n} h_{m n} f_{n}$.

Time-invariant linear systems constitute a subclass of the class of linear systems 1 . They are characterized by kernels of the form $h\left(t, t^{\prime}\right)=\eta\left(t-t^{\prime} ;\right.$, so that the output is the convolution of the input $f(t)$ with $\eta(t)$ :

\footnotetext{
I This is the standard terminology of signal theory where $t$ usually denotes time. In Fourier optics, where the independent variable (usually $\boldsymbol{x}$ ) denotes spatial position, such systems are called spaceinvariant systems. More generally, they might be referred to as shift-invariant systems.
}

$g(t)=\int_{-\infty}^{\infty} \eta\left(t-t^{\prime}\right) f\left(t^{\prime}\right) \mathrm{d} t^{\prime} \equiv \eta(t) * f(t)$

Bacause Eq. (3) is a convolution, it describes a simpis multiplication in the Fourier domain. Hence, convolution-type operations are also referred to as "linear filtering" operations. The operation expressed by Eq. (1) is far more general than that expressed by Eq. (3), as evidenced by the fact that $h\left(t, t^{\prime}\right)$ has two degrees of freedom opposed to the one degree of freedom of $\eta(t)$.

There are several useful operations which cannot be performed by time-invariant systems. Firstly, timeinvariant systems cannot perform any operation which introduces frequency components that did not already exist in the input pulse ${ }^{2}$. For example, we can compress a pulse down to a width $r$ only if it contains frequency components at $\pm \tau^{-1}$ to begin with. A timevarying system of the form given by $\mathrm{Eq}$. (1), on

\footnotetext{
${ }^{2}$ This can be proven by taking the Fourier transferm of both sides of Eq. (3).
} 
the other hand (like an electro-optic phase modulator followed by linear dispersion), can compress a pulse regardless of its original frequency content. A second example of an operation that requires a timevariant system is rearrangement of the bits within a bit sequence such as $b_{3} b_{2} b_{1} b_{0}$ to obtain, for instance, $b_{0} b_{2} b_{3} b_{1}$ (bit-slot rearrangement). Thirdly, chirp-like distortions (i.e., a signal component with a frequency varying linearly in time) cannot be filtered out by a time-invariant process. This is because such distorting signals have a large spread in both the time and frequency domains and thus will have a large overlap with the desired signal in both domains. Recalling that a time-invariant filter corresponds to a mask in the frequency domain, it will not be possible to successfully separate the distortion from the signal. Such distortions can be eliminated using fractional Fourier domain filters, which are time-variant operations [1]. The latter example should not be confused with chirp compensation [2] such as used in femtosecond pulse compression or to avoid broadening of data bits due to spectral dispersion in fiber communications systems, which is only concerned with retiming of chirped signals, but not with filtering out chirped distortions.

Unlike time-variant operations, space-variant operations are easy to implement. For example, an object can easily be compressed (i.e., demagnified) using lenses, and spatial slot interchange can be handled using matrix-vector multipliers [3]. Many researchers have pointed out the similarities between spatial and temporal optical systems [4-6]. This suggests that spatial processing techniques may be used to solve the equivalent temporal processing task. In this paper, we prove that Eq. (1) cannot be implemented in the time-domain with passive linear components only. However, we propose an all-optical system involving time-space conversions and nonlinear components that realizes Eq. (1) in a relatively simple way.

When two-dimensional functions of space are concerned, optical implementation of the two-dimensional spatial version of Eq. (3) is realized using the " $4 f$ " system (Fig. 1a) [7]. The "4f" performs the convolution operation of Eq. (3) by linear multiplicative filtering in the Fourier plane of the setup. If we are willing to use a two-dimensional optical system to implement (the spatial version of) Eq. (1) in only one dimension, this is possible using an architecture known as a matrix-vector multiplier [3]. The name of this a)

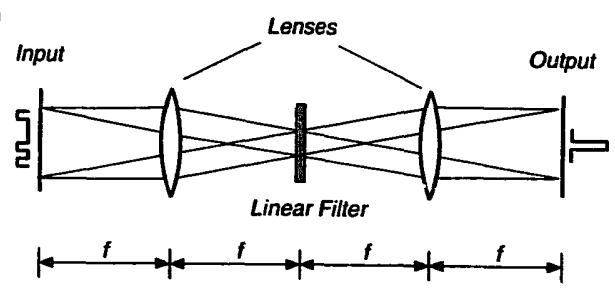

b)

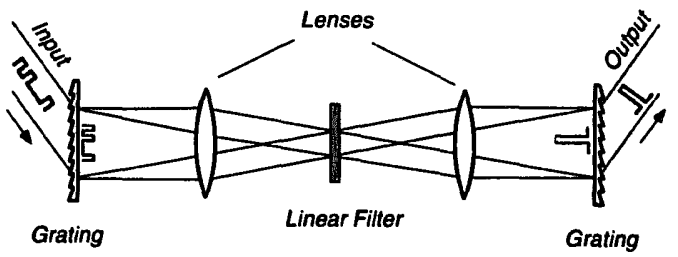

Fig. 1. (a) Space-invariant spatial processor in the common "4f" configuration. (b) Time-invariant temporal pulse processor. Free-space diffraction sections of (a) are replaced by gratings.

architecture is known by this name since the discrete version of Eq. (1) is a matrix-vector product.

In principle, the temporal counterpart of the " $4 \mathrm{f}$ " system (as well as that of a more general class of systems described below), can be realized by substituting temporal modulators for the filters, temporal chirp modulators for the lenses (also referred to as "time lenses"), and dispersive devices (fibers or gratings) for the free-space sections $[4,5]$. With these replacements, the mathematical analogy is exact - however, the experimental and technological requirements are much different. What is achieved in the spatial case with a simple lens, requires a sophisticated modulator in the temporal case. It turns out that all temporal operations described by Eq. (3) can be performed without active elements such as modulators. Of particular interest is the configuration shown in Fig. 1b, which is obtained by introducing gratings at the input and output planes of Fig. 1a $[8,9]$. Although transmission gratings are shown for clarity in the figure, regular reflection gratings are normally used. This system separates the different temporal frequency components spatially in the Fourier plane, so that a spatial filter can be used to perform temporal spectral filtering.

Let us try to understand how the system of Fig. 1b works. It is possible to show $[8,10,6]$ that if $f(t)$ denotes the input pulse, the waveform immediately after the grating is given by $[10,6]$ 


$$
f(t-\beta x / c),
$$

where $\beta=\lambda /\left(d \cos \theta_{\text {out }}\right)$, with $\theta_{\text {out }}$ being the diffraction angle of the grating, $d$ the pitch of the grating and $\lambda$ the center wavelength of the input pulse.

Although the above result can be found in the references, it will be instructive to show how it is derived. Assume that the grating lies in the $z=0$ plane with its rulings parallel to the $y$-axis. A monochromatic plane wave of frequency $\omega$ with incidence angle $\theta_{\text {in }}$ may be expressed as $\exp \left(i \omega \sin \theta_{i n} x / c\right)$. Its first-order diffraction angle $\theta_{\text {out }}$ will satisfy the grating equation

$\sin \theta_{\text {out }}(\omega)=\sin \theta_{\text {in }}-2 \pi r / \omega d$.

An input pulse $f(t)$ vill consist of a continuum of frequency componenis centered around some central frequency $\omega_{0}$. The diffraction angle $\theta_{\text {out }}(\omega)$ for any of these components can be expanded as

$\theta_{\text {out }}(\omega)=\theta_{\text {out }}\left(\omega_{0}\right)+\left.\left(\omega-\omega_{0}\right) \frac{\mathrm{d} \theta_{\text {out }}(\omega)}{\mathrm{d} \omega}\right|_{\omega=\omega_{0}}+\cdots$,

which can be put in the form

$$
\begin{aligned}
& \theta_{\text {out }}(\omega)-\theta_{\text {out }}\left(\omega_{0}\right) \simeq \beta \Omega / \omega_{0}, \\
& \left.\beta \equiv \omega_{0} \frac{\mathrm{d} \theta_{\text {our }}(\omega)}{\mathrm{d} \omega}\right|_{\omega \text { a } 00},
\end{aligned}
$$

where we have defined $\Omega \equiv \omega-\omega_{0}$. The grating equation can be used to evaluate $\beta$;

$$
\beta=\frac{\lambda}{d \cos \theta_{\text {out }}\left(\omega_{0}\right)} \text {. }
$$

Thus, if we define a broken optical axis in the conventional manner such that it makes the angle $\theta_{\text {in }}$ with the $z$-axis on the left, and makes the angle $\theta_{\text {out }}\left(\omega_{0}\right)$ with the $z$-axis on the right, we see that the effect of the grating can be captured by a transmittance function $T(x, \Omega)$ of the form

$$
T(x, \Omega)=\exp (i \beta \Omega x / c)
$$

for the component with frequency $\Omega$. (This is most easily seen by recalling the transmittance function $\exp (2 \pi \Delta \theta x / \lambda)$ of a prism with deflection angle $\Delta \theta$.) An arbitrary pulse $f(t)$ can be expressed as a linear superposition of frequency components of the form $\exp (-i \Omega t)$ and each component multiplied by
$T(x, \Omega)$. By using an elementary Fourier transform theorem, the effect of this space-dependent phase factor applied in the temporal frequency domain, is a space-dependent time-shift in the time domain, as given by Eq. (4).

If we freeze time in Eq. (4), we see that the temporal pulse has been mapped onto a spatial waveform. Thus a time-to-space conversion has taken place. Now, the " $4 \mathrm{f}$ " setup, which is the spatial realization of Eq. (3), can be used to perform the desired operation. This is followed by a space-to-time conversion, converting the waveform $g(t-\beta x / c)$ immediately before the output grating to the temporal output $g(t)$. Naively, this may seem to suggest that we have a generic method of utilizing any spatial optical system for realizing the corresponding temporal system. The reason that this is not true (as proven mathematically below), is that the waveform $f(t-\beta x / c)$ is not steady but moving at a velocity $c / \beta$. This does not matter in Fig. $1 b$, since the time-dependent shift translates into a time-dependent phase factor in the filter (Fourier) plane, which is not altered as we pass through the filter and once again appears as the time-dependent shift of $g(t-\beta x / c)$ just before the output grating.

The longest temporal waveform that can be converted into a spatial representation is given by the inverse spectral resolution of the grating setup, which is determined by the grating constant and the number of lines illuminated by the input beam. This time window is typically on the order of tens to hundreds of picoseconds, depending on the grating constant and beam configuration [6].

Despite its flexibility, the most general operation which can be performed by such a linear temporal system which is passive (in the sense of not having any time-varying components such as modulators), is of the form of Eq. (3). Proof: Let us initially assume that the more general Eq. (1) holds. Let the system be excited with a Dirac delta function $\delta(t)$, and the corresponding output be denoted by $g_{0}(t)$, which is seen to be equal to $h(t, 0)$. Now, if instead we had excited the system with a delayed delta function $\delta(t-$ $\left.t_{0}\right)$, and if the system has no time-varying components, the output in this case must be $g_{0}\left(t-t_{0}\right)$, which is seen to be equal to $h\left(t, t_{0}\right)$. Comparing the outputs in both cases, we obtain $h\left(t-t_{0}, 0\right)=h\left(t, t_{0}\right)$. Since this is true for all $t_{0}$, it follows that $h\left(t, t^{\prime}\right)$ can be expressed in the form $h\left(t-t^{\prime}, 0\right)$, which can be defined 


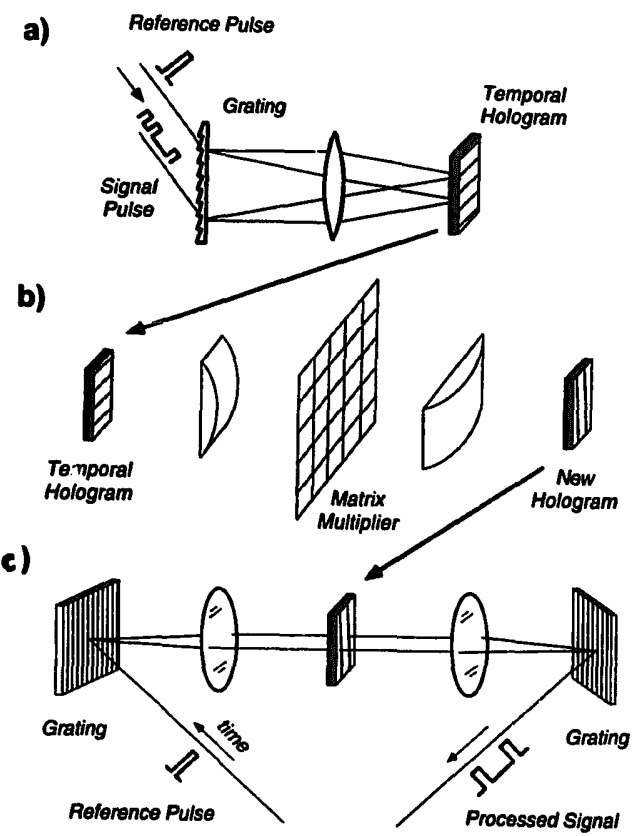

Fig. 2. Temporal matrix-vector processor. The temporal data is first fixed in a temporal hologram (a), the matrix-vector operation is then performed on this hologram directly in the Fourier domain (b), creating a new hologram. Finally, the information in the second hologram is transferred back into the time-domain by reading it out in a temporal pulse processor (c). Although the three steps are shown separately for clarity, they can be combined using beam splitters and combiners.

as the $\eta\left(t-t^{\prime}\right)$ appearing in Eq. (3). The converse of the above theorem is also true: any system of the form of Eq. (3) can be implemented using passive components.

Since a temporal optical system realizing Eq. (1) is not possible with passive linear elements only, we propose a relatively simple implementation involving nonlinear elements. The idea involves (i) writing a spectral hologram of the temporal waveform [6], (ii) performing a matrix-vector multiplication in the Fourier domain on the information stored in the hologram. The output of the matrix-vector multiplier is recorded in a second hologram, which (iii) is then read out in a temporal " $4 \mathrm{f}$ " system to convert the processed data back into the time domain [11]. The optical systems to perform these operations are shown in Fig. 2. Although the three parts of the system are drawn separately for clarity, they can be combined so that the system can be operated in real time, just limited by the response time of the holographic material.

Fig. 2a shows the setup for writing a hologram of the temporal signal [6]. In addition to the signal pulse, a short reference pulse is diffracted from the input grating, and the interference fringes between the signal and reference spectra in the Fourier plane are recorded in a real-time holographic material. Again, transmission gratings are shown for clarity only. Because both signal and reference pulses surf across the grating with the same speed, the interference fringes in the spectrum plane are stationary and do not move as the pulses "surf" across the grating. Mathematically, upon spatial Fourier transformation with a " $2 \mathrm{f}$ " setup, the spatio-temporal waveform $f(t-\beta x / c)$ becomes $\propto$ $F\left(c k_{x} / \beta\right) \exp \left(-i k_{x} c t / \beta\right)$, where $k_{x}$ denotes spatial frequency, and $F(\Omega)$ is the Fourier transform of $f(t)$. (Of course, this spatial frequency $k_{x}$ is represented physically along a spatial coordinate, say $\hat{x}$, in the Fourier plane with $k_{x}=\hat{x} /(\lambda \times$ lens focal length $)$. $)$ If the reference pulse is approximated by a Dirac delta $\delta(t)$ whose Fourier transform is unity, the recorded hologram becomes

$$
\begin{aligned}
\mid \exp & \left(-i k_{x} c t / \beta\right)+\left.F\left(c k_{x} / \beta\right) \exp \left(-i k_{x} c t / \beta\right)\right|^{2} \\
\quad & =\left|1+F\left(c k_{x} / \beta\right)\right|^{2} \\
& =1+\left|F\left(c k_{x} / \beta\right)\right|^{2}+F^{*}\left(c k_{x} / \beta\right)+F\left(c k_{x} / \beta\right),
\end{aligned}
$$

where the last term, representing the Fourier transform of $f(t)$, but mapped into the spatial frequency domain, is the one that is of interest to us.

Eq. (1) can now be implemented using a matrixvector multiplier, as shown schematically in Fig. $2 b$ [3]. Because the matrix operation is performed on the Fourier hologram rather than in real space, we have to construct a mask that performs the matrix-vector operation in Fourier space rather than in real space. We can rewrite Eq. (1) in the Fourier domain:

$$
G(\Omega)=\int_{-\infty}^{\infty} H\left(\Omega, \Omega^{\prime}\right) F\left(\Omega^{\prime}\right) \mathrm{d} \Omega^{\prime},
$$

or

$G\left(c k_{x} / \beta\right) \propto \int_{-\infty}^{\infty} H\left(c k_{x} / \beta, c k_{x}^{\prime} / \beta\right) F\left(c k_{x}^{\prime} / \beta\right) \mathrm{d} k_{x}^{\prime}$,

where capital letters denote Fourier transforms. $H\left(\Omega, \Omega^{\prime}\right)$ is related to $h\left(t, t^{\prime}\right)$ through a similar- 
ity transformation, since the Fourier transform is a unitary operation:

$$
\begin{aligned}
& H\left(\Omega, \Omega^{\prime}\right) \\
& \quad=\frac{1}{2 \pi} \int_{-\infty}^{\infty} \int_{-\infty}^{\infty} h\left(t, t^{\prime}\right) \exp \left[i\left(\Omega t-\Omega^{\prime} t^{\prime}\right)\right] \mathrm{d} t \mathrm{~d} t^{\prime}
\end{aligned}
$$

Now, using the hologram $F\left(c k_{x} / \beta\right)$ as a spatial input mask (by illuminating it with a plane wave), we can record a mask corresponding to $G\left(c k_{x} / \beta\right)$ by using the matrix-vector multiplier. The spatial output of the matrix multiplier then has to be converted back into the time domain. This is accomplished by reading out the new hologram $G\left(c k_{x} / \beta\right)$ with a short reconstruction pulse in the temporal " $4 \mathrm{f}$ " setup (Fig. $2 \mathrm{c}$ ). This final operation creates $g(t-\beta x / c)$ just before the output grating, which is then converted into $g(t)$ by the grating. Mathematically, the reconstruction pulse $\delta(t)$ is spatially Fourier transformed into $\exp \left(-i k_{x} c t / \beta\right)$ as before, which upon multiplication with $G\left(c k_{x} / \beta\right)$ gives

$\exp \left(-i k_{x} c t / \beta\right) G\left(c k_{x} / \beta\right)$,

which upon spatial Fourier transformation in the second half of the system is converted into $g(t-\beta x / c)$. The second grating then converts this into the temporal pulse $g(t)$.

As mentioned before, the three setups in Fig. 2 can be cascaded to produce a real-time optical system. This requires that the holographic material can be recorded and reau out simultaneously in real time. A system such as the one in Fig. $2 a$ has been demonstrated with photorefractive multi-quantum-wells (MQW) employed as a holographic material [6]. This material showed a $3 \%$ diffraction efficiency and a $2 \mu s$ response time. With some sacrifices on the diffraction efficiency, the intrinsic response time of the MQW material can be as short as 10 ps [12], thus allowing processing speeds of tens of $\mathrm{Gb} / \mathrm{s}$.

The proposed system is not the only way to perform general linear operations in the time domain. Matrix-vector multiplication can in principle also be performed using time lenses and dispersive devices. (Time lenses are components which multiply a time function $f(t)$ with a chirp function $\exp \left[i\right.$ (const) $\left.t^{2}\right]$, in analogy with a spatial lens. Dispersive devices can be used to convolve a time function with a chirp function, in analogy with free-space propagation [4,5].) However, in such a system, the matrix-vector operation still has to take place in the time-domain with all its limitations and problems. One often overlooked feature of a temporal processor based on time-lenses is that in these optical systems, time is converted into frequency [13]. Thus a spectrometer can spatially disperse these different time (frequency) slots, and one can perform the matrix-vector multiplication in the spatial domain. Unlike in our proposal, a second synchronized reference pulse is not required, but the phase modulation of the time lens needs to be synchronized to the input pulse pattern, which may be very hard to do. On the other hand, the quadratic phase modulation of a time lens can also be obtained by upconversion or four-wave-mixing (FWM) with a square chirped pulse [14], avoiding the use of an electro-optic phase modulator. The efficiency of the frequency conversion process would determine the usefulness of this approach in real applications.

The benefit of discussions with David A. B. Miller of Bell Laboratories, Holmdel, New Jersey, USA is gratefully acknowledged.

\section{References}

[1] H.M. Ozaktas, B. Barshan, D. Mendlovic and L. Onural, J. Opt. Soc. Am. A 11 (1994) 547.

[2] O.E. Martinez. IEEE J. Quantuin Electron. 23 (1987) 59.

(3) R.A. Heinz, J.O. Artman and S.H. Lee, Appl. Optics 9 (1970).

|4| B. Kolner, IEEE J. Quantum Electron. 30 (1994) 1951.

[5] A.W. Lohmann and D. Mendlovic, Appl. Optics 31 (1992) 6212.

[6] M.C. Nuss, M. Li, T.H. Chiu and A.M. Weiner, Optics Lett. 19 (1994) 664.

17] J.W. Goodman. Introduction to Fourier Optics (McGrawHill, New York, 1968).

[8] A.M. Weiner, J.P. Heritage and E.M. Kirschner, J. Opt. Soc. Am. B 5 (1988) 1563.

[9] A.M. Weiner and D.E. Leaird, Optics Lett. 15 (1990) 51.

[10] Yu.T. Mazurenko, Appl. Physics B 50 (1990) 101.

[11] M.C. Nuss and R.L. Morrison, Optics Lett. 20 (1995) 740.

[12] J.A. Cavailles, D.A.B. Miller, J.E. Cunningham, P.L.K. Wa and A. Miller, IEEE J. Quantum Electron. 28 (1992) 2486.

[13] M.T. Kauffman, W.C. Banyai, A.A. Godil and D.M. Bloom, Appl. Phys. Lett. 64 (1994) 270.

[14] C.V. Bennet, R.P. Scott and B.H. Kolner, Appl. Phys. Lett. 65 (1994) 2513. 